\title{
The value of pulmonary nodule diameter and consolidation/tumor rate in the prediction of lymph node metastasis in early-stage (cT1NOM0) lung adenocarcinoma
}

\author{
Weipeng Shao ${ }^{1}$, Zhaohua Zhang ${ }^{1}$, Zhan Liu ${ }^{1}$, Zhenrong Zhang ${ }^{2}$, Hongliang Sun ${ }^{3}$, Xiaowei Wang ${ }^{4}$, \\ Hongxiang Feng ${ }^{2}$, Chaoyang Liang ${ }^{2}$, Deruo Liu ${ }^{1}$ \\ ${ }^{1}$ Department of General Thoracic Surgery, Peking University China-Japan Friendship School of Clinical Medicine, Beijing, China; ${ }^{2}$ Department of \\ General Thoracic Surgery, China-Japan Friendship Hospital, Beijing, China; ${ }^{3}$ Department of Radiology, China-Japan Friendship Hospital, Beijing, \\ China; ${ }^{4}$ Department of Pathology, China-Japan Friendship Hospital, Beijing, China \\ Contributions: (I) Conception and design: W Shao, Z Zhang, D Liu; (II) Administrative support: D Liu; (III) Provision of study materials or patients: \\ All authors; (IV) Collection and assembly of data: W Shao, Z Zhang; (V) Data analysis and interpretation: W Shao, Z Zhang, H Feng; (VI) \\ Manuscript writing: All authors; (VII) Final approval of manuscript: All authors. \\ Correspondence to: Deruo Liu. Department of General Thoracic Surgery, Peking University China-Japan Friendship School of Clinical Medicine, No. \\ 2, Yinghua, East Rd, Beijing 100029, China. Email: deruoliu@163.com; Zhenrong Zhang. Department of General Thoracic Surgery, China-Japan \\ Friendship Hospital, No. 2, Yinghua, East Rd, Beijing 100029, China. Email: doctor_zzr@126.com.
}

\begin{abstract}
Background: We aimed to determine whether the use of pulmonary nodule diameter and CTR predicts lymph nodes (LNs) metastasis for early-stage (cT1N0M0) lung adenocarcinoma.

Methods: We retrospectively analyzed 433 consecutive patients who underwent therapeutic surgical resection in our hospital. Information about age, sex, history of malignancy, smoking index, high-resolution computed tomography (HRCT) imaging information, pathologic findings, and status of LNs metastasis were collected.

Results: A total of 433 patients were included 277 women and 156 men, with a median age of 58.09 \pm 9.41 years. On univariate and multivariate analysis, visceral pleural invasion (VPI) $(\mathrm{P}=0.005)$, the diameter of nodule measured by postoperative pathology $(\mathrm{DP})(\mathrm{P}=0.011)$, the largest axial diameter of the lesion on the mediastinal window $(\mathrm{DM})(\mathrm{P}<0.001)$, the ratio of the maximum diameter of consolidation relative to the maximum tumor diameter from the lung window (CTR) $(\mathrm{P}=0.01)$, and total dissected $\mathrm{LNs}$ number $(\mathrm{P}=0.005)$ categories were independent facto for LNs metastasis. The receiver operating characteristic (ROC) curve showed that DM $\geq 11.81 \mathrm{~cm}$, or CTR $\geq 79.50 \%$, or VPI indicated LNs metastasis. LNs metastasis patients could be better predicted by a total dissected LNs number with a cutoff point of 13.5 for lung cancer.

Conclusions: VPI, DP, DM, CTR, and total dissected LNs number categories were independent factors for LNs metastasis. If $\mathrm{DM} \geq 11.81 \mathrm{~cm}$, or CTR $\geq 79.50 \%$, or VPI systemic lymphadenectomy was recommended. We suggested $14 \mathrm{LNs}$ as the cut point for the evaluation LNs examination.
\end{abstract}

Keywords: Lung cancer; lymph node (LN) metastasis; CT images; consolidation/tumor rate

Submitted Jul 17, 2020. Accepted for publication Nov 27, 2020.

doi: $10.21037 /$ tcr-20-2548

View this article at: http://dx.doi.org/10.21037/tcr-20-2548

\section{Introduction}

Lung cancer is the leading cause of cancer-related mortality worldwide, with approximately $85 \%$ of patients having nonsmall cell lung cancer (NSCLC) (1). Surgery is still the first choice of treatment for localized NSCLC if the patient's physical condition is feasible. However, the postresection 5 -year survival rate is only $50 \%$ to $60 \%$ (2). The primary tumor, lymph nodes (LNs), and the metastasis (TNM) 
staging system remain the most important determinant of outcome (3). Because prognosis of lung cancer is directly proportional to the presence of LNs metastasis, accurate LNs assessment is crucial in determining treatment. Could radical systematic LNs dissection or sampling lead to a different result on patients with cIA NSCLC? Up to now, although there have been several prospective controlled studies published, they had never reached a consensus (46). The pulmonary nodule diameter and consolidation/ tumor ratio (CTR) is a well-established radiological parameters for identifying pathologic noninvasive tumors or invasive tumors on CT images of the chest $(7,8)$. However, there are limited studies on the relationship between CT imaging information and LNs metastasis. Therefore, we aimed to determine whether the use of pulmonary nodule diameter and CTR predicts LNs metastasis for earlystage (cT1N0M0) lung adenocarcinoma. We present the following article in accordance with the STARD reporting checklist (available at http://dx.doi.org/10.21037/tcr-202548).

\section{Methods}

The study was conducted in accordance with the Declaration of Helsinki (as revised in 2013). The study was approved by the institutional ethics board of China-Japan Friendship Hospital (No. 2018-13-K08) and Consent of patients was waived because it was a retrospective study.

\section{Data collection}

We retrospectively analyzed 433 consecutive patients who underwent therapeutic surgical resection in China-Japan Friendship Hospital between February 2011 and February 2019. We included patients with the following criteria: (I) received lobectomy and sublobectomy without any preoperative chemoradiotherapy; (II) was preoperatively staged as clinical stage IA (T1N0M0, tumor size $\leq 3 \mathrm{~cm}$ ); (III) was pathologically confirmed with peripherally located primary lung adenocarcinoma (outer one-third of the hemithorax); or (IV) underwent systematic LNs dissection or sampling based on the European Society of Thoracic Surgeons criteria (9).

Chest images were obtained using 256-detector row CT scanners (Philips Brilliance), 64-detector row CT scanners (Siemens SOMA-TOM Sensation), and a 16-detector row CT scanner (Toshiba Aquilion). High-resolution images of the tumors were acquired using the following parameters:
$120 \mathrm{kV}$ and auto-exposure control; collimation, $0.6-1.25 \mathrm{~mm}$; pitch, $0.9-0.984 ; 0.4-0.5 \mathrm{~s}$ per rotation; reconstructed interval, $1.25-1.5 \mathrm{~mm}$; pixel resolution, $512 \times 512$; a field of view, $20 \mathrm{~cm}$; and lung window settings (level $=-500 /$ width $=1,500 \mathrm{HU}$ ) with high spatial frequency algorithm and mediastinal window settings $($ level $=40 /$ width $=320 \mathrm{HU}$ ) with soft tissue algorithm.

Information about age, sex, history of malignancy, smoking index, high-resolution computed tomography (HRCT) imaging information, pathologic findings, and status of LNs metastasis were collected.

The HRCT imaging information contains the following: (I) the diameter of the tumor (T) was defined as the largest axial diameter of the lesion on the lung window setting; (II) the diameter of consolidation $(\mathrm{C})$ on the axial image on the lung window setting was measured, and consolidation was defined as an area of increased opacification that completely obscured the underlying bronchial structures and vascular markings; (III) the largest axial diameter of the lesion on the mediastinal window (DM) setting was also measured; (IV) Diameter of nodule measured by postoperative pathology (DP); (V) the ratio of the maximum diameter of consolidation relative to the maximum tumor diameter from the lung window (CTR). Status of LNs metastasis contains the following: (I) total dissected mediastinal LNs number and metastasis rate; (II) total dissected N1 (hilar or intrapulmonary) LNs number and metastasis rate. CT images were independently evaluated by a radiologist with more than 10 years' experience in thoracic imaging diagnosis and a thoracic surgeon with more than 10 years' experience in diagnosis and treatment of thoracic disease. The clinicopathological characteristics of the patients were not informed in advance to avoid any potential bias. Length measurements were carried out by manual cursor method in the Picture Archiving and Communication System (PACS).

All patients were staged according to the eighth edition of the TNM staging system for lung cancer (3).

\section{Statistical analysis}

Categoric variables were expressed as percentages and evaluated with $\lambda 2$ or Fisher's exact test. Continuous data were presented as mean $\pm \mathrm{SD}$ and were compared using the ANOVA, two-sample Student $t$ test and nonparametric tests. Receiver-operating characteristic (ROC) curves were plotted to assess the predictive value of LNs metastasis in early-stage lung adenocarcinoma. All P-values were reported by 2 -sided analyses, and the statistical significance level was set at less 
than 0.05 . Statistical analysis was performed with SPSS 24.0 software (SPSS, Inc., Chicago, IL, USA).

\section{Results}

\section{Patient characteristics}

A total of 433 patients were included 277 women and 156 men, with a median age of $58.09 \pm 9.41$ years. The median tumor size was $16.28 \pm 6.06 \mathrm{~mm}$. Among these patients, $353(81.5 \%)$ patients denied smoking. There were 396 patients who received lobectomy and 37 patients received sublobectomy. We grouped these patients into 3 groups according to tumor size $(\mathrm{cT} 1 \mathrm{a} \leq 1 \mathrm{~cm} ; \mathrm{cT} 1 \mathrm{~b}>1 \mathrm{~cm}$ but $\leq 2 \mathrm{~cm}$; cT1c $>2 \mathrm{~cm}$ but $\leq 3 \mathrm{~cm})$. The number of cT1a, cT1b, and cT1c patients was 82,234 , and 117 , respectively. The mean total number of dissected N1 LNs of cT1a, cT1b, and cT1c patients were $5.74 \pm 3.79,6.35 \pm 3.56$, and $7.12 \pm 3.45$, respectively. The rate of $\mathrm{N} 1 \mathrm{LN}$ metastasis was $0,8.12 \%$, and $23.90 \%$, respectively. Postoperative pathology confirmed that $99(22.9 \%)$ patients had visceral pleural invasion (VPI), which led to the stage from cT1 to pT2a, 51 occurred in cT1c group. There was significant difference of age $(\mathrm{P}<0.001)$, smoking index $(\mathrm{P}=0.011)$, surgery $(\mathrm{P}=0.001)$, VPI $(\mathrm{P}<0.001)$, DP $(\mathrm{P}<0.001)$, total dissected $\mathrm{LNs}$ number $(\mathrm{P}<0.001)(11.45 \pm 5.47,13.49 \pm 6.90,15.26 \pm 5.90$, respectively $)$, radiographic feature [including $\mathrm{T}(\mathrm{P}<0.001), \mathrm{C}(\mathrm{P}<0.001)$, $\mathrm{DM}(\mathrm{P}<0.001)$, and CTR $(\mathrm{P}<0.001)]$ among the 3 groups. Detailed patient characteristics were listed in Table 1.

\section{Univariate and multivariate analysis for LNs metastasis}

According to the occurrence of LNs metastasis, we continued to divide all patients into the $\mathrm{N} 0$ group and $\mathrm{N} 1 / 2$ group. In the univariate analysis for survival, sex $(\mathrm{P}=0.003)$, smoking index $(\mathrm{P}=0.002)$, VPI $(\mathrm{P}<0.001)$, cT $(\mathrm{P}<0.001)$, total dissected LNs number $(\mathrm{P}<0.001)$, DP $(\mathrm{P}<0.001)$, and radiographic feature (including $\mathrm{T}, \mathrm{C}, \mathrm{DM}$, and CTR) $(\mathrm{P}<0.001)$ categories were identified as prognostic factors after surgical resection (Table 2). On multivariate analysis, VPI $(\mathrm{P}=0.005), \mathrm{DP}(\mathrm{P}=0.011), \mathrm{DM}(\mathrm{P}<0.001)$, CTR $(\mathrm{P}=0.01)$, and total dissected $\mathrm{LNs}$ number $(\mathrm{P}=0.005)$ categories were independent facto for LNs metastasis (Table 3).

\section{Associations between clinical-pathological parameters and LNs metastasis}

Figure 1 showed the receiver-operating characteristic curves. The diagonal line indicated an area under the curve of 0.5 , equivalent to the measurement having no predictive value. The area under the curve for CTR was $0.911(\mathrm{P}=0.014,95 \%$ CI, 0.883-0.939) (Figure 1). If CTR was used to predict the LNs metastasis and assuming a threshold of $79.50 \%$ for defining high risk for LNs metastasis, the sensitivity and specificity were $98.3 \%$ and $75.3 \%$, respectively. The area under the curve for DM was also $0.911(\mathrm{P}=0.014,95 \%$ CI, 0.883-0.939) (Figure 1). If DM was used to predict the LNs metastasis and assuming a threshold of $11.81 \mathrm{~cm}$ for defining high risk for LNs metastasis, the sensitivity and specificity were $91.7 \%$ and $80.9 \%$, respectively. The area under the curve for the total dissected LNs number was 0.704 (Figure 1). The area under the curve for total dissected LNs number was 0.704 ( $\mathrm{P}=0.035$, 95\% CI, 0.635 $0.773)$ and $\mathrm{DP}$ was $0.726(\mathrm{P}=0.031,95 \% \mathrm{CI}, 0.665-0.786)$ (Figure 1). The area under the curve was between $0.7-0.9$, which means the diagnostic value was moderate. If the total dissected LNs number was used to predict the LNs

Table 1 Patient characteristics

\begin{tabular}{|c|c|c|c|c|}
\hline Variable & T1a $(n=82)$ & $\mathrm{T} 1 \mathrm{~b}(\mathrm{n}=234)$ & $\mathrm{T} 1 \mathrm{c}(\mathrm{n}=117)$ & $\mathrm{P}$ \\
\hline Male & 21 & 93 & 42 & 0.072 \\
\hline Female & 67 & 141 & 75 & \\
\hline Age, y & $54.35 \pm 10.10$ & $59.16 \pm 9.58$ & $58.59 \pm 7.87$ & $<0.001$ \\
\hline No & 79 & 221 & 116 & 0.057 \\
\hline Yes & 3 & 13 & 1 & \\
\hline
\end{tabular}

Table 1 (continued) 
Table 1 (continued)

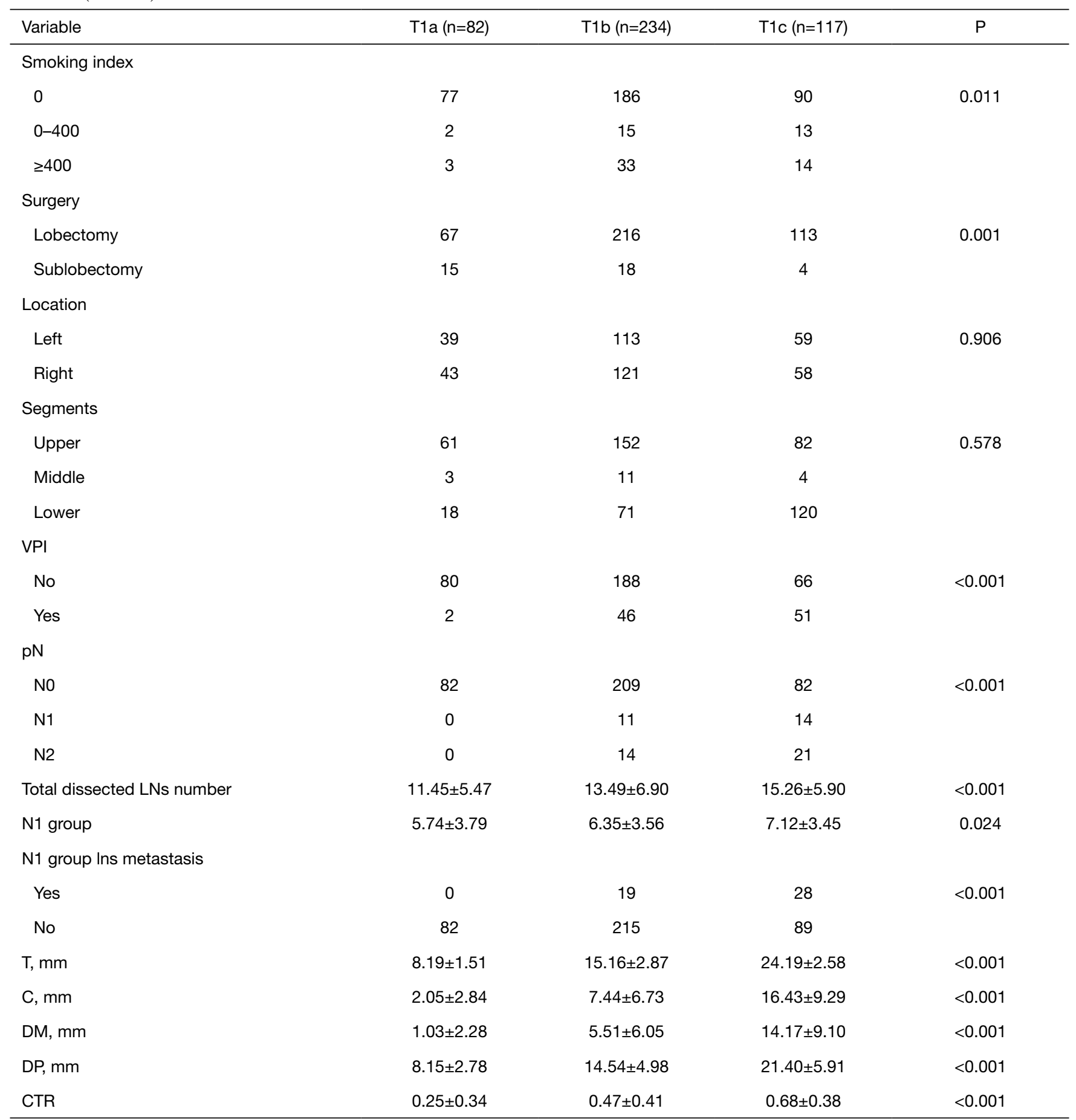

VPI, visceral pleural invasion; pN, conventional pathologically lymph nodes; LN, lymph nodes; T, the largest axial diameter of the lesion on the lung window setting; $\mathrm{C}$, the diameter of consolidation on the axial image on the lung window setting; DM, the largest axial diameter of the lesion on the mediastinal window setting; DP, diameter of nodule measured by postoperative pathology; CTR, the ratio of the maximum diameter of consolidation relative to the maximum tumor diameter from the lung window. 
Table 2 Univariate analysis for LNs metastasis

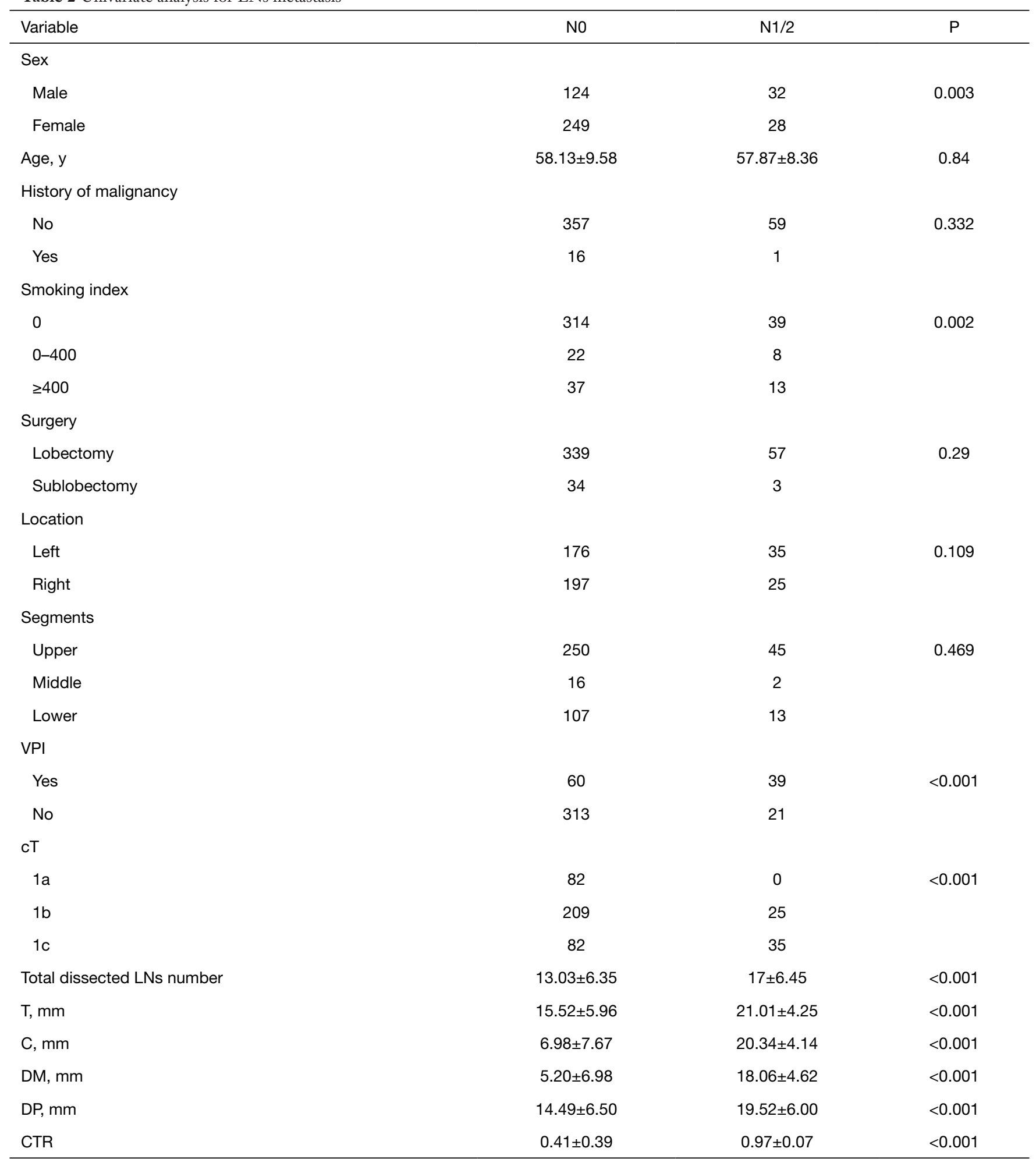

LN, lymph nodes; VPI, visceral pleural invasion; T, the largest axial diameter of the lesion on the lung window setting; C, the diameter of consolidation on the axial image on the lung window setting; DM, the largest axial diameter of the lesion on the mediastinal window setting; DP, diameter of nodule measured by postoperative pathology; CTR, the ratio of the maximum diameter of consolidation relative to the maximum tumor diameter from the lung window. 
Table 3 Multivariate analysis for LNs metastasis

\begin{tabular}{|c|c|c|c|}
\hline Variable & OR & $95 \% \mathrm{Cl}$ & $P$ \\
\hline $\mathrm{DP}$ & 0.37 & $0.17-0.8$ & 0.011 \\
\hline DM & 1.19 & $1.08-1.3$ & $<0.001$ \\
\hline CTR & $28,044.61$ & $75.63-103,99,940.44$ & 0.01 \\
\hline
\end{tabular}

LN, lymph nodes; VPI, visceral pleural invasion; DM, the largest axial diameter of the lesion on the mediastinal window setting; DP, diameter of nodule measured by postoperative pathology; CTR, the ratio of the maximum diameter of consolidation relative to the maximum tumor diameter from the lung window. OR: Odds ratio.

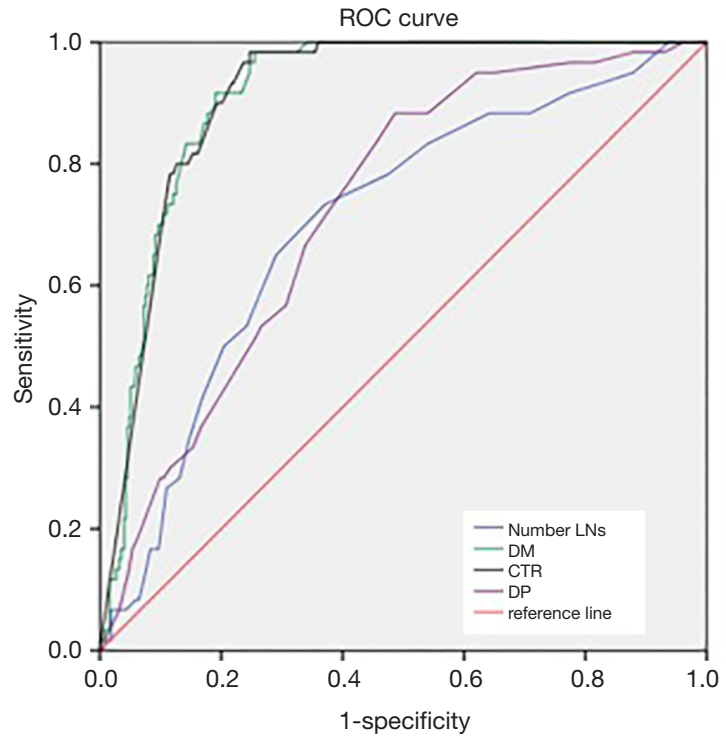

Figure 1 Receiver-operating characteristic curves. Reference line: (AUC 0.5); Number LNs: baseline total dissected LNs number (AUC 0.704); DP: baseline diameter of nodule measured by postoperative pathology (AUC 0.726); DM: baseline the largest axial diameter of the lesion on the mediastinal window setting (AUC 0.911); CTR: baseline the ratio of the maximum diameter of consolidation relative to the maximum tumor diameter from the lung window (AUC 0.911). Diagonal segments are produced by ties.

metastasis, and assuming a threshold of 13.5 for defining high risk for LNs metastasis, the sensitivity and specificity were $73.3 \%$ and $62.9 \%$, respectively.

\section{Discussion}

\section{The tumor size and LNs metastasis}

Pulmonary nodules were further divided into solid, ground- glass, and part-solid categories. The detection of smallsized lung cancer was increasing with the development of low dose CT (LDCT), however, the preoperative evaluation of LNs status with CT scan was remaining to be difficult mainly because many cancer positive nodes of normal size could be seen on pathological examination especially in adenocarcinoma cases. It has been proved that the maximum diameter of the nodule was associated with LNs metastasis $(10,11)$. In our study, we have chosen the diameters measured in various ways (including $\mathrm{T}, \mathrm{C}$, DM, and DP). According to the largest axial diameter of the lesion on the lung window setting, IASLC Lung Cancer Staging Project results show that cT1 tumors could be divided into three subgroups based on the best cut points identified by the running log-rank analysis (3). Pathological N1 LNs metastasis in cT1a, cT1b, and cT1c group was $0 \%, 4.70 \%, 11.97 \%$, respectively. Pathological $\mathrm{N} 2$ LNs metastasis in cT1a, cT1b, and cT1c group was $0 \%$, $5.98 \%, 17.95 \%$, respectively. The rate of LNs metastasis increases with the increase of T. Many early-stage lung cancer showed ground-glass opacity (GGO) nodules on chest CT. Generally, GGO on a chest CT was considered an indication of noninvasive or minimally invasive tumors (12). Part-solid GGOs tended to have increased malignant invasiveness as the solid portion increases (13). Saji et al. believed that the predictive values of the solid tumor size visualized on HRCT especially in the mediastinal window for pathologic high-grade malignancy and prognosis in lung adenocarcinoma were greater than those of whole tumor size (14). In our multivariate analysis, DP and DM were independent prognostic factors for LNs metastasis. DP was difficult to obtain before an operation, so the value of prediction was poor. We suggested that the DM be used to predict LNs metastasis in cases of lung adenocarcinoma with a GGO component visualized on 
HRCT. According to the ROC curve and Youden index, we calculated that the best cutoff point was $11.81 \mathrm{~cm}$. We recommended systematic LN dissection if DM was greater than $11.81 \mathrm{~cm}$ and the pathological type was adenocarcinoma.

\section{CTR and LNs metastasis}

Previous studies have revealed that a CTR of 0.5 or less was an important cutoff value for predicting pathologic invasiveness in lung adenocarcinoma $(15,16)$. Similarly, we also noted that there was no LNs metastasis in patients with a cutoff point of less than $50 \%$. The Japan Clinical Oncology Group (JCOG) 0201 study revealed that CTR of 0.25 or less was an important cutoff value for predicting pathologic noninvasiveness in lung adenocarcinoma of $2.0 \mathrm{~cm}$ or less (17). A lower CTR corresponded with less aggressive behavior, whereas a higher CTR indicated a more aggressive tumor. There were further studies on CTR and LNs metastasis. Heineman et al. reported that LNs metastasis occurred in $14.9 \%$ of patients with clinical stage I NSCLC after anatomical resection (18). In our research, CTR was an independent factor for LNs metastasis. According to the ROC curve and the Youden index, an LNs metastasis patient could be predicted by a CTR with a cutoff point of $79.50 \%$ and a specificity of 98.3\% (95\% CI, 0.883-0.939) for peripherally located primary lung adenocarcinoma $\leq 3.0 \mathrm{~cm}$ in size. As a result, $79.50 \%$ cutoff point showed the highest specificity, although its sensitivity was relatively low. However, Moon et al. believed that patients with a CTR $\geq 0.61$ had LNs metastasis (19). The reason for such a big difference in cutoff point might be the fact that they only included clinical N0 patients, and we had no limit on clinical N staging. The CTR $\geq 79.50 \%$ provided clinically safe criteria to identify LNs metastasis.

\section{Total dissected LNs number and LNs metastasis}

In the current lung cancer TNM staging system, the anatomic extent of LNs metastases was the only factor used to define the N category of TNM (20). Some studies have demonstrated that the number of positive LNs was an important prognostic factor for resected NSCLC (21). According to ACOSOG Z0030 results, National Comprehensive Cancer Network (NCCN) recommends systematic lymphadenectomy or sampling for lung cancer patients, with at least $12 \mathrm{LNs}$ removed (4). Liang et al. recommended $16 \mathrm{LNs}$ as the cutoff point for evaluating the quality of $\mathrm{LN}$ examination or prognostic stratification postoperatively for patients with declared node-negative disease (22). In our study, the total dissected LNs number was an independent factor for LNs metastasis. According to the ROC curve and the Youden index, LNs metastasis patients could be better predicted by a total dissected LNs number with a cutoff point of 13.5 for lung cancer. ACCP only recommended that the minimum requirements for accurate nodal staging must include the removal of at least six LNs from hilar and mediastinal stations (5). Complete resection including 14 or more LNs provided more information for determining LNs metastasis and TNM classification.

\section{VPI and LNs metastasis}

The presence of VPI reflected a poor prognosis in resected patients with lung cancer (23). Primary tumor cT1 with VPI was upgraded to pT2a in the 8th edition of the TNM classification system for patients with lung cancer (3). In our analysis, VPI was an independent factor for LNs metastasis. The VPI rate was only $2.43 \%$ in cT1a group, but up to $43.59 \%$ in cT1c group. Moon et al. believed that VPI (HR 2.901, $\mathrm{P}=0.005$ ) was a predictor of LNs metastasis in clinical N0 peripheral NSCLC with nodules size $\leq 3 \mathrm{~cm}$ (24). Ding et al. also found that VPI (OR 48.37, $\mathrm{P}<0.001)$ was significantly associated with LNs metastasis in clinical stage IA NSCLC (25). If VPI was found during the operation, lymphadenectomy should be taken and all visible LNs should be removed.

Our study still suffered from several limitations. First, as a retrospective study, it was vulnerable to various sources of deviation that might not be identified and controlled. Second, this was a single institutional study with a limited sample size and thus calls for more evidence from further prospective studies (including a larger series of patients).

\section{Conclusions}

Under the eighth edition TNM classification, with the increase of tumor diameter, the probability of LNs metastasis increases. On univariate and multivariate analysis, VPI, DP, DM, CTR, and total dissected LNs number categories were independent factors for LNs metastasis. If $\mathrm{DM} \geq 11.81 \mathrm{~cm}$, or CTR $\geq 79.50 \%$, or VPI, systemic lymphadenectomy was recommended. We suggested 14 LNs as the cut point for the evaluation of the quality of 
postoperative LNs examination.

\section{Acknowledgments}

Funding: None.

\section{Footnote}

Reporting Checklist: The authors have completed the STARD reporting checklist. Available at http://dx.doi.org/10.21037/ tcr-20-2548

Data Sharing Statement: Available at http://dx.doi. org/10.21037/tcr-20-2548

Peer Review File: Available at http://dx.doi.org/10.21037/tcr20-2548

Conflicts of Interest: All authors have completed the ICMJE uniform disclosure form (available at http://dx.doi. org/10.21037/tcr-20-2548). The authors have no conflicts of interest to declare.

Ethical Statement: The authors are accountable for all aspects of the work in ensuring that questions related to the accuracy or integrity of any part of the work are appropriately investigated and resolved. The study was conducted in accordance with the Declaration of Helsinki (as revised in 2013). The study was approved by the institutional ethics board of China-Japan Friendship Hospital (No. 2018-13-K08) and Consent of patients was waived because it was a retrospective study.

Open Access Statement: This is an Open Access article distributed in accordance with the Creative Commons Attribution-NonCommercial-NoDerivs 4.0 International License (CC BY-NC-ND 4.0), which permits the noncommercial replication and distribution of the article with the strict proviso that no changes or edits are made and the original work is properly cited (including links to both the formal publication through the relevant DOI and the license). See: https://creativecommons.org/licenses/by-nc-nd/4.0/.

\section{References}

1. Bray F, Ferlay J, Soerjomataram I, et al. Global cancer statistics 2018: GLOBOCAN estimates of incidence and mortality worldwide for 36 cancers in 185 countries. CA
Cancer J Clin 2018;68:394-424.

2. Woodard GA, Jones KD, Jablons DM. Lung Cancer Staging and Prognosis. Cancer Treat Res 2016;170:47-75.

3. Goldstraw P, Chansky K, Crowley J, et al. The IASLC Lung Cancer Staging Project: Proposals for Revision of the TNM Stage Groupings in the Forthcoming (Eighth) Edition of the TNM Classification for Lung Cancer. J Thorac Oncol 2016;11:39-51.

4. Darling GE, Allen MS, Decker PA, et al. Randomized trial of mediastinal lymph node sampling versus complete lymphadenectomy during pulmonary resection in the patient with N0 or N1 (less than hilar) non-small cell carcinoma: results of the American College of Surgery Oncology Group Z0030 Trial. J Thorac Cardiovasc Surg 2011;141:662-70.

5. Detterbeck FC, Postmus PE, Tanoue LT. The stage classification of lung cancer: Diagnosis and management of lung cancer, 3rd ed: American College of Chest Physicians evidence-based clinical practice guidelines. Chest 2013;143:e191S-e210S.

6. Hishida T, Miyaoka E, Yokoi K, et al. Lobe-Specific Nodal Dissection for Clinical Stage I and II NSCLC: Japanese Multi-Institutional Retrospective Study Using a Propensity Score Analysis. J Thorac Oncol 2016;11:1529-37.

7. Travis WD, Brambilla E, Nicholson AG, et al. The 2015 World Health Organization Classification of Lung Tumors: Impact of Genetic, Clinical and Radiologic Advances Since the 2004 Classification. J Thorac Oncol 2015;10:1243-60.

8. Hattori A, Matsunaga T, Takamochi K, et al. Importance of Ground Glass Opacity Component in Clinical Stage IA Radiologic Invasive Lung Cancer. Ann Thorac Surg 2017;104:313-20.

9. De Leyn P, Lardinois D, Van Schil PE, et al. ESTS guidelines for preoperative lymph node staging for non-small cell lung cancer. Eur J Cardiothorac Surg 2007;32:1-8.

10. Wang YX, Li BS, Huang W, et al. Pattern of lymph node metastases and its implication in radiotherapeutic clinical target volume in patients with non-small-cell lung cancer: a study of 2062 cases. Br J Radiol 2015;88:20140288.

11. Shafazand S, Gould MK. A clinical prediction rule to estimate the probability of mediastinal metastasis in patients with non-small cell lung cancer. J Thorac Oncol 2006;1:953-9.

12. Westaway DD, Toon CW, Farzin M, et al. The International Association for the Study of Lung Cancer/ American Thoracic Society/European Respiratory Society 
grading system has limited prognostic significance in advanced resected pulmonary adenocarcinoma. Pathology 2013;45:553-8.

13. Wilshire CL, Louie BE, Manning KA, et al. Radiologic Evaluation of Small Lepidic Adenocarcinomas to Guide Decision Making in Surgical Resection. Ann Thorac Surg 2015;100:979-88.

14. Saji H, Matsubayashi J, Akata S, et al. Correlation between whole tumor size and solid component size on highresolution computed tomography in the prediction of the degree of pathologic malignancy and the prognostic outcome in primary lung adenocarcinoma. Acta Radiol 2015;56:1187-95.

15. Zhou Q, Suzuki K, Anami Y, et al. Clinicopathologic features in resected subcentimeter lung cancer--status of lymph node metastases. Interact Cardiovasc Thorac Surg 2010;10:53-7.

16. Suzuki K, Kusumoto M, Watanabe S, et al. Radiologic classification of small adenocarcinoma of the lung: radiologic-pathologic correlation and its prognostic impact. Ann Thorac Surg 2006;81:413-9.

17. Suzuki K, Koike T, Asakawa T, et al. A prospective radiological study of thin-section computed tomography to predict pathological noninvasiveness in peripheral clinical IA lung cancer (Japan Clinical Oncology Group 0201). J Thorac Oncol 2011;6:751-6.

18. Heineman DJ, Ten BM, Daniels JM, et al. Clinical Staging of Stage I Non-Small Cell Lung Cancer in the Netherlands-Need for Improvement in an Era With Expanding Nonsurgical Treatment Options: Data From

Cite this article as: Shao $W$, Zhang Z, Liu Z, Zhang Z, Sun H, Wang X, Feng H, Liang C, Liu D. The value of pulmonary nodule diameter and consolidation/tumor rate in the prediction of lymph node metastasis in early-stage (cT1N0M0) lung adenocarcinoma. Transl Cancer Res 2021;10(1):38-46. doi: $10.21037 /$ tcr-20-2548 the Dutch Lung Surgery Audit. Ann Thorac Surg 2016;102:1615-21.

19. Moon Y, Park JK, Lee KY, et al. Consolidation/Tumor Ratio on Chest Computed Tomography as Predictor of Postoperative Nodal Upstaging in Clinical T1N0 Lung Cancer. World J Surg 2018;42:2872-8.

20. Detterbeck FC, Boffa DJ, Tanoue LT, et al. Details and difficulties regarding the new lung cancer staging system. Chest 2010;137:1172-80.

21. Lee JG, Lee CY, Park IK, et al. Number of metastatic lymph nodes in resected non-small cell lung cancer predicts patient survival. Ann Thorac Surg 2008;85:211-5.

22. Liang W, He J, Shen Y, et al. Impact of Examined Lymph Node Count on Precise Staging and Long-Term Survival of Resected Non-Small-Cell Lung Cancer: A Population Study of the US SEER Database and a Chinese MultiInstitutional Registry. J Clin Oncol 2017;35:1162-70.

23. Seok Y, Lee E. Visceral Pleural Invasion Is a Significant Prognostic Factor in Patients with Partly Solid Lung Adenocarcinoma Sized $30 \mathrm{~mm}$ or Smaller. Thorac Cardiovasc Surg 2018;66:150-5.

24. Moon Y, Choi SY, Park JK, et al. Risk Factors for Occult Lymph Node Metastasis in Peripheral Non-Small Cell Lung Cancer with Invasive Component Size $3 \mathrm{~cm}$ or Less. World J Surg 2020;44:1658-65.

25. Ding N, Mao Y, Gao S, et al. Predictors of lymph node metastasis and possible selective lymph node dissection in clinical stage IA non-small cell lung cancer. J Thorac Dis 2018;10:4061-8. 